\title{
Asymptotic Preserving Time-Discretization of Optimal Control Problems for the Goldstein-Taylor Model
}

\author{
Giacomo Albi ${ }^{1}$, Michael Herty ${ }^{2}$, \\ Christian Jörres ${ }^{2}$, and Lorenzo Pareschi ${ }^{1}$
}

Bericht Nr. 371

Juli 2013

Key words: IMEX Runge-Kutta methods, optimal boundary control, hyperbolic conservation laws, asymptotic analysis

AMS Subject Classifications: 35Q93, 65M06, 35L50

Institut für Geometrie und Praktische Mathematik RWTH Aachen

Templergraben 55, D-52056 Aachen (Germany)

1 University of Ferrara, Department of Mathematics and Computer Science, Via Machiavelli 35, I-44121 Ferrara, Italy, e-mail: \{giacomo.albi, lorenzo.pareschi\}@unife.it

2 RWTH Aachen University, Templergraben 55, D-52065 Aachen, Germany,

e-mail: \{herty,joerres\}@igpm.rwth-aachen.de 


\title{
Asymptotic Preserving time-discretization of optimal control problems for the Goldstein-Taylor model
}

\author{
G. Albi ${ }^{* 1}$, M. Herty ${ }^{\dagger 2}$, C. Jörres ${ }^{\ddagger 2}$, and L. Pareschi ${ }^{\S 1}$ \\ ${ }^{1}$ University of Ferrara, Department of Mathematics and Computer Science, Via \\ Machiavelli 35, I-44121 Ferrara, ITALY \\ ${ }^{2}$ RWTH Aachen University, Templergraben 55, D-52065 Aachen, GERMANY
}

July 31, 2013

\begin{abstract}
We consider the development of implicit-explicit time integration schemes for optimal control problems governed by the Goldstein-Taylor model. In the diffusive scaling this model is a hyperbolic approximation to the heat equation. We investigate the relation of time integration schemes and the formal Chapman-Enskog type limiting procedure. For the class of stiffly accurate implicit-explicit Runge-Kutta methods (IMEX) the discrete optimality system also provides a stable numerical method for optimal control problems governed by the heat equation. Numerical examples illustrate the expected behavior.
\end{abstract}

Keywords: IMEX Runge-Kutta methods, optimal boundary control, hyperbolic conservation laws, asymptotic analysis

\section{Introduction}

We are interested in numerical methods for time discretization of optimal control problems of type (1.1). The construction of such methods for control problems involving differential equations has been an intensive field of research recently [9, 19, 20, 28, 34, 43]. Applications

\footnotetext{
*giacomo.albi@unife.it

†herty@igpm.rwth-aachen.de

¥joerres@igpm.rwth-aachen.de

${ }^{\S}$ lorenzo.pareschi@unife.it
} 
of such methods can be found in several disciplines, form aerospace and mechanical engineering to the life sciences. In particular, many applications involves systems of differential equations of the form

$$
y^{\prime}(t)=f(y(t), t)+\frac{1}{\varepsilon} g(y(t), t),
$$

where $f$ and $g$, eventually obtained as suitable finite-difference or finite-element approximations of spatial derivatives, induce considerably different time scales indicated by the small parameter $\varepsilon>0$ in the previous equation. Therefore, to avoid fully implicit integrators, it is highly desirable to have a combination of implicit and explicit (IMEX) discretization terms to resolve stiff and non-stiff dynamics accordingly. For Runge-Kutta methods such schemes have been studied in $[1,11,18,31,32,35,36]$.

Control problems with respect to IMEX methods have been investigated also in $[29,30]$ in the case of fixed positive value of $\varepsilon>0$. Among the most relevant examples for IMEX scheme are the time discretization of hyperbolic balance laws and kinetic equations. As discussed in $[32,36]$ the construction of such methods imply new difficulties due to the appearance of coupled order conditions and to the possible loss of accuracy close to stiff regimes $\varepsilon \ll \Delta t$ and $\Delta t$ being the time discretization of the numerical scheme. In contrary to the existing work $[29,30]$ we focus here on optimal control problems where the time integration schemes also allow a accurate resolution in the stiff regime. As a prototype example including already the major difficulties for such methods we choose the GoldsteinTaylor model (1.3). This equation already contains several ingredients typical to linear kinetic transport models and serves as a prototype and test case for numerical integration schemes. The model describes the time evolution of two particle densities $f^{+}(x, t)$ and $f^{-}(x, t)$, with $x \in \Omega \subset \mathbb{R}$ and $t \in \mathbb{R}^{+}$, where $f^{+}(x, t)$ (respectively $f^{-}(x, t)$ ) denotes the density of particles at time $t>0$ traveling along a straight line with velocity $+c$ (respectively $-c$ ). The particle may change with rate $\sigma$ the direction. The differential model can be written as

$$
\begin{aligned}
& f_{t}^{+}+c f_{x}^{+}=\sigma\left(f^{-}-f^{+}\right), \\
& f_{t}^{-}-c f_{x}^{-}=\sigma\left(f^{+}-f^{-}\right) .
\end{aligned}
$$

Introducing the macroscopic variables

$$
\rho=f^{+}+f^{-}, \quad j=c\left(f^{+}-f^{-}\right)
$$

we obtain the equivalent form

$$
\begin{aligned}
\rho_{t}+j_{x} & =0, \\
j_{t}+c^{2} \rho_{x} & =-2 \sigma j .
\end{aligned}
$$

We introduce a linear quadratic optimal control problem subject to a relaxed hyperbolic system of balance laws. Let $\Omega=[0,1]$, terminal time $T>0$, regularisation parameter $\nu \geq 0$ 
and let $u(t)$ be the control. The function $\rho_{d}(x)$ is a desired state. To simplify notation we set $c^{2}=2 \sigma=1 / \varepsilon^{2}$ and $\varepsilon>0$ is the non-negative relaxation parameter.

The optimization problem then reads

$$
\min J(\rho, u)=\frac{1}{2} \int_{0}^{1}\left(\rho(x, T)-\rho_{d}(x)\right)^{2} d x+\frac{\nu}{2} \int_{0}^{T} u^{2}(t) d t
$$

subject to

$$
\begin{array}{rlrl}
\rho_{t}+j_{x} & =0, & \\
j_{t}+\frac{1}{\varepsilon^{2}} \rho_{x} & =-\frac{1}{\varepsilon^{2}} j . & & \\
\rho(x, 0) & =\rho_{0}, & & j(x, 0)=j_{0} \\
j(0, t) & =0, & j(1, t)-\rho(1, t)=-u(t)
\end{array}
$$

Further, we set box constraints for the control

$$
u_{l}(t) \leq u(t) \leq u_{r}(t)
$$

In the limit case $\varepsilon \rightarrow 0,(1.5 \mathrm{~b})$ formally yields

$$
j(x, t)=-\rho_{x}(x, t) .
$$

Plugging this into (1.5a) yields the heat equation

$$
\rho_{t}=\rho_{x x}
$$

and the optimal control problem (1.4) - (1.5) reduces to a problem studied for example in [39]. Obviously, we expect a similar behavior for a numerical discretization. This property, called asymptotic preserving, has been investigated for the simulation of Goldstein-Taylor like models in $[11,18,31]$ but has not yet been studied in the context of control problems. The paper is organized as follows. In Section 2 we introduce the temporal discretization of problem (1.5) and describe in detail the resulting semi-discretized optimal control problems. We investigate which numerical integration schemes yield a stable approximation to the resulting optimality conditions. In the third section we show how to provide a stable discretization scheme in the parabolic regime by introducing a splitting and applying the formal Chapman-Enskog type limiting procedure. In Section 4 we present numerical results on the several implicit explicit Runge-Kutta methods (IMEX) schemes for the limiting problem as well as on an example taken from [39]. Definitions for properties of the IMEX schemes are collected for convenience in the appendix A. 


\section{The semi-discretized problem}

We are interested to derive a numerical time integration scheme which allows to treat the optimal control problem (1.4)-(1.5) for all values of $\varepsilon \in[0,1]$, including in particular the limit case $\varepsilon=0$. Therefore, we leave a side the treatment of the discretization of the spatial variable $x$ as well as theoretical aspects of the differentiability of solutions $(\rho, J)$ of equation (1.5). We remark that the semigroup generated by a nonlinear hyperbolic conservation/balance law is generically non-differentiable in $L^{1}$ even in the scalar onedimensional (1-D) case. More details on the differential structure of solutions are found in $[12,13,14]$, on convergence results for first-order numerical schemes and scalar conservation laws are found in $[4,17,24,26,27,41]$ Numerical methods for the optimal control problems of scalar hyperbolic equations have been discussed in [3, 23, 25, 42]. In [21, 22], the adjoint equation has been discretized using a Lax-Friedrichs-type scheme, obtained by including conditions along shocks and modifying the Lax-Friedrichs numerical viscosity. Convergence of the modified Lax-Friedrichs scheme has been rigorously proved in the case of a smooth convex flux function. Convergence results have also been obtained in [40] for the class of schemes satisfying the one-sided Lipschitz condition (OSLC) and in [3] for a first-order implicit-explicit finite-volume method. To the best of our knowledge there does not exists a convergence theory for spatial discretization of control problems subject to hyperbolic systems with source terms so far.

In view of the previous discussion the interest is on the availability of suitable timeintegration schemes for the arising optimal control problem. We consider therefore a semidiscretized problem in time. We further skip the spatial dependence whenever the intention is clear. The system (1.5a) consists of a stiff and a non-stiff part we employ diagonal implicit explicit Runge-Kutta methods (IMEX). Convergence order of such schemes for positive $\varepsilon$ and the property of symplecticity has been analysed in [30]. In the following we briefly review IMEX methods and discuss a splitting [11] in order to also resolve efficiently the stiff limiting problem $(\varepsilon=0)$.

An $s$-stage IMEX Runge-Kutta method is characterized by the $s \times s$ matrices $\tilde{A}, A$ and vectors $\tilde{c}, c, \tilde{b}, b \in \mathbb{R}^{s}$, represented by the double Butcher tableau:

$$
\begin{array}{l|cl}
\text { Explicit: } \quad & \tilde{c} & \tilde{A} \\
\hline & \tilde{b}^{T}
\end{array} \quad \text { Implicit: } \quad \begin{array}{c|c}
c & A \\
\hline & b^{T}
\end{array}
$$

We refer to the appendix A for further definitions and examples of IMEX RK schemes. Applying an IMEX time-discretization to the Goldstein-Taylor model (1.5) yields in the limit $\varepsilon=0$ an explicit numerical scheme for the heat equation [11]. This is only stable provided the parabolic CFL condition $\Delta t \approx \Delta x^{2}$ holds true. This is highly undesirable and therefore, a splitting has been introduced such that also in the limit $\varepsilon=0$ an implicit 
discretization of the heat equation can be obtained. We rewrite (1.5a) as

$$
\rho_{t}=-\overbrace{\left(j+\mu \rho_{x}\right)_{x}}^{\text {explicit }}+\overbrace{\left(\mu \rho_{x x}\right)}^{\text {implicit }}
$$

where $\mu=\mu(\varepsilon) \geq 0$ is such that $\mu(0)=1$ and leave equation (1.5b) unchanged. Within an IMEX time discretization we treat explicitly the first term and implicitly the second term as indicated in (2.1). It remains to discuss the choice of $\mu$ in equation 2.1 depending on $\varepsilon$. Using formal Chapman-Enkog expansion for this choice, presented in section B, we observe that in the diffusive limit $\varepsilon=0$ the term $j+\mu \rho_{x}$ vanishes.

Combining the previous computations we state the semi-discretized problem for an $s$-stage IMEX scheme. Introduce a temporal grid of size $\Delta t$ and $N$ equally spaced grid points $t_{n}$ such that $T=\Delta t N$ and $t_{1}=0$. Let $\rho^{n}=\rho\left(t_{n}, \cdot\right), j^{n}=j\left(t_{n}, \cdot\right), \mathbf{e}=(1, \ldots, 1) \in \mathbb{R}^{s}$ and denote by $\mathbf{R}=\left(R_{\ell}(\cdot)\right)_{\ell=1}^{s}$ the $s$ stage variables and similarly for $\mathbf{J}$. For notational simplicity we discretize the control on the same temporal grid $u^{n}=u\left(t_{n}\right)$. However, this is not necessary for the derived results and other approaches can be used. We prescribe boundary conditions in the case $\varepsilon>0$ as follows: Since in the limit $\varepsilon=0$ we obtain $j(t, x)=-\rho_{x}(t, x)$ we add $j^{n}(1)=-\rho_{x}^{n}(1)$ and $j^{n}(0)=-\rho_{x}^{n}(0)$ as boundary conditions. Further let $\mathcal{M}=\operatorname{diag}\left(\mu_{l}\right) \in \mathbb{R}^{s \times s}$ define the values of $\mu_{l}$ for the levels $l=1, \ldots, s$.

Then, the semi-discretization of problem (1.5) reads

$$
\begin{aligned}
\min & \frac{1}{2} \int_{0}^{1}\left(\rho^{N}(x)-\rho_{d}(x)\right)^{2} d x+\Delta t \frac{\nu}{2} \sum_{n=1}^{N}\left(u^{n}\right)^{2}, \\
\mathbf{R} & =\rho^{n} \mathbf{e}-\Delta t \tilde{A}\left(\partial_{x} \mathbf{J}+\mathcal{M} \partial_{x x}^{2} \mathbf{R}\right)+\Delta t A\left(\mathcal{M} \partial_{x x}^{2} \mathbf{R}\right), \\
\varepsilon^{2} \mathbf{J} & =\varepsilon^{2} j^{n} \mathbf{e}-\Delta t A\left(\partial_{x} \mathbf{R}+\mathbf{J}\right), \\
\rho^{n+1} & =\rho^{n}-\Delta t \tilde{b}^{T}\left(\partial_{x} \mathbf{J}+\mathcal{M} \partial_{x x}^{2} \mathbf{R}\right)+\Delta t b^{T}\left(\mathcal{M} \partial_{x x}^{2} \mathbf{R}\right), \\
\varepsilon^{2} j^{n+1} & =\varepsilon^{2} j^{n}-\Delta t b^{T}\left(\partial_{x} \mathbf{R}+\mathbf{J}\right), \\
\rho^{1} & =\rho_{0} \quad j^{1}=j_{0}, \\
j^{n}(0) & =0, \quad j^{n}(1)-\rho^{n}(1)=-u^{n}, \\
j^{n}(0) & =-\rho_{x}^{n}(0), \quad j^{n}(1)=-\rho^{n}(1)_{x} .
\end{aligned}
$$

Using formal computations we derive the (adjoint) equations (2.3) for the Lagrange multipliers $\left(p^{n}, q^{n}\right)_{n=1}^{N}$ and the corresponding stage variables $\mathbf{P}, \mathbf{Q}$ with $\mathbf{P}=\left(P_{\ell}(\cdot)\right)_{\ell=1}^{s}$, $P_{\ell} \in \mathbb{R}^{s}$ and $\mathbf{Q}$ respectively. 


$$
\begin{aligned}
p^{n}= & p^{n+1}+\mathbf{e}^{T} \mathbf{P}, \quad \rho^{N}-\rho_{d}-p^{N}=0, \\
\varepsilon^{2} q^{n}= & \varepsilon^{2} q^{n+1}+\varepsilon^{2} \mathbf{e}^{T} \mathbf{Q}, \quad \varepsilon^{2} q^{N}=0, \\
\mathbf{P}= & \Delta t\left(\partial_{x}\left(A^{T} \mathbf{Q}\right)+\partial_{x} q^{n+1} b\right)-\Delta t \mathcal{M}\left(\partial_{x x}^{2}\left(\tilde{A}^{T} \mathbf{P}\right)+\partial_{x x}^{2} p^{n+1} \tilde{b}\right) \\
& +\Delta t \mathcal{M}\left(\partial_{x x}^{2}\left(A^{T} \mathbf{P}\right)+\partial_{x x}^{2} p^{n+1} b\right), \\
\varepsilon^{2} \mathbf{Q}= & -\Delta t\left(A^{T} \mathbf{Q}+q^{n+1} b\right)+\Delta t\left(\partial_{x}\left(\tilde{A}^{T} \mathbf{P}\right)+\partial_{x} p^{n+1} \tilde{b}\right) .
\end{aligned}
$$

We obtain boundary conditions for (2.3) as

$$
q^{n}(0)=0, \quad q^{n}(1)+p^{n}(1)=0, \quad q^{n}(0)=p_{x}^{n}(0) \quad \text { and } \quad q^{n}(1)=p_{x}^{n}(1) .
$$

Furthermore, we consider under the assumption of using a type A scheme (we leave on purpose a definitions of these scheme in appendix A) the limit case $\varepsilon=0$ of the optimal control problem (1.5). Note that for $\varepsilon=0$ we have $\mathcal{M}=\mathrm{Id}$. The semi-discretized problem is

$$
\begin{aligned}
\min & \frac{1}{2} \int_{0}^{1}\left(\rho^{N}(x)-\rho_{d}(x)\right)^{2} d x+\Delta t \frac{\nu}{2} \sum_{n=1}^{N}\left(u^{n}\right)^{2} \\
\mathbf{R} & =\rho^{n} \mathbf{e}-\Delta t \tilde{A}\left(\partial_{x} \mathbf{J}+\partial_{x x}^{2} \mathbf{R}\right)+\Delta t A\left(\partial_{x x}^{2} \mathbf{R}\right) \\
\mathbf{J} & =-\partial_{x} \mathbf{R} \\
\rho^{n+1} & =\rho^{n}-\Delta t \tilde{b}^{T}\left(\partial_{x} \mathbf{J}+\partial_{x x}^{2} \mathbf{R}\right)+\Delta t b^{T}\left(\partial_{x x}^{2} \mathbf{R}\right) \\
\rho^{1} & =\rho_{0} \quad \rho_{x}^{n}(0)=0, \quad \rho_{x}^{n}(1)+\rho^{n}(1)=u^{n},
\end{aligned}
$$

and the corresponding adjoint equations are given by (2.6).

$$
\begin{aligned}
p^{n}= & p^{n+1}+\mathbf{e}^{T} \mathbf{P}, \quad \rho^{N}-\rho_{d}-p^{N}=0, \\
\mathbf{P}= & \partial_{x} \overline{\mathbf{Q}}-\Delta t\left(\partial_{x x}^{2}\left(\tilde{A}^{T} \mathbf{P}\right)+\partial_{x x}^{2} p^{n+1} \tilde{b}\right) \\
& +\Delta t\left(\partial_{x x}^{2}\left(A^{T} \mathbf{P}\right)+\partial_{x x}^{2} p^{n+1} b\right) \\
\overline{\mathbf{Q}}= & \Delta t\left(\partial_{x}\left(\tilde{A}^{T} \mathbf{P}\right)+\partial_{x} p^{n+1} \tilde{b}\right)
\end{aligned}
$$

We obtain boundary conditions for (2.6) as

$$
p_{x}^{n}(1)+p^{n}(1)=0, \quad \text { and } \quad p_{x}^{n}(0)=0 .
$$

The relation between the limiting problem and the small $\varepsilon$ limit of the adjoint equations (2.3) and (2.6) is summarized in the following Lemma. 
Lemma 2.1. If the IMEX Runge Kutta method is implicit stiffly accurate (ISA) and of type $A$, then the $\varepsilon=0$ limit of (2.3) is given by

$$
\begin{aligned}
p^{n}= & \mathbf{e}^{t} \mathbf{P}, \rho^{N}-\rho_{d}-p^{N}=0, \quad q^{n}=0, q^{N}=0, \\
\mathbf{P}= & p^{n+1} \mathbf{e}_{s}+\Delta t \partial_{x}\left(A^{T} \mathbf{Q}\right) \\
& -\Delta t\left(\partial_{x x}^{2}\left(\tilde{A}^{T} \mathbf{P}\right)-\partial_{x x}^{2}\left(A^{T} \mathbf{P}\right)\right)-\Delta t\left(\tilde{b}^{T}-\mathbf{e}_{s}^{T} \tilde{A}\right) \partial_{x x}^{2} p^{n+1} \mathbf{e} \\
0= & -\Delta t\left(A^{T} \mathbf{Q}-\partial_{x}\left(\tilde{A}^{T} \mathbf{P}\right)\right)+\Delta t\left(\tilde{b}^{T}-\mathbf{e}_{s} \tilde{A}\right) \partial_{x} p^{n+1} \mathbf{e}^{T}
\end{aligned}
$$

Further, there exists a linear variable transformation such that a solution to (2.8) is equivalent to a solution of the adjoint equation (2.6) of Problem (2.5) for $\varepsilon=0$.

Proof. In the case of implicit stiffly accurateness the IMEX scheme simplifies to

$$
\begin{aligned}
\mathbf{R} & =\rho^{n} \mathbf{e}-\Delta t \tilde{A}\left(\partial_{x} \mathbf{J}+\mathcal{M} \partial_{x x}^{2} \mathbf{R}\right)+\Delta t A\left(\mathcal{M} \partial_{x x}^{2} \mathbf{R}\right) \\
\varepsilon^{2} \mathbf{J} & =\varepsilon^{2} j^{n} \mathbf{e}-\Delta t A\left(\partial_{x} \mathbf{R}+\mathbf{J}\right) \\
\rho^{n+1} & =\mathbf{e}_{s}^{T} \mathbf{R}-\Delta t\left(\tilde{b}^{T}-\mathbf{e}_{s}^{T} \tilde{A}\right)\left(\partial_{x} \mathbf{J}+\mathcal{M} \partial_{x x}^{2} \mathbf{R}\right), \quad j^{n+1}=\mathbf{e}_{s}^{T} \mathbf{J}
\end{aligned}
$$

and the corresponding adjoint equations are given by

$$
\begin{aligned}
p^{n}= & \mathbf{e}^{t} \mathbf{P}, q^{n}=\varepsilon^{2} \mathbf{e}^{T} \mathbf{Q}, \quad \rho^{N}-\rho_{d}-p^{N}=0, \quad \varepsilon^{2} q^{N}=0 \\
\mathbf{P}= & p^{n+1} \mathbf{e}_{s}+\Delta t \partial_{x}\left(A^{T} \mathbf{Q}\right) \\
& -\Delta t \mathcal{M}\left(\partial_{x x}^{2}\left(\tilde{A}^{T} \mathbf{P}\right)-\partial_{x x}^{2}\left(A^{T} \mathbf{P}\right)\right)-\Delta t\left(\tilde{b}^{T}-\mathbf{e}_{s}^{T} \tilde{A}\right) \partial_{x x}^{2} p^{n+1} \mathcal{M} \mathbf{e} \\
\varepsilon^{2} \mathbf{Q}= & q^{n+1} \mathbf{e}_{s}-\Delta t\left(A^{T} \mathbf{Q}-\partial_{x}\left(\tilde{A}^{T} \mathbf{P}\right)\right)+\Delta t\left(\tilde{b}^{T}-\mathbf{e}_{s}^{T} \tilde{A}\right) \partial_{x} p^{n+1} \mathbf{e}
\end{aligned}
$$

Since $\mathcal{M}=$ Id in the limit $\varepsilon=0$ we obtain the adjoint equations (2.8). Introducing the transformation

$$
\overline{\mathbf{Q}}=\Delta t A^{T} \mathbf{Q}
$$

and proceeding yields from (2.8) the system (2.10).

$$
\begin{aligned}
p^{n}= & \mathbf{e}^{T} \mathbf{P}, q^{n}=0, \quad \rho^{N}-\rho_{d}-p^{N}=0, q^{N}=0, \\
\mathbf{P}= & p^{n+1} \mathbf{e}_{s}+\partial_{x} \overline{\mathbf{Q}} \\
& -\Delta t\left(\partial_{x x}^{2}\left(\tilde{A}^{T} \mathbf{P}\right)-\partial_{x x}^{2}\left(A^{T} \mathbf{P}\right)\right)-\Delta t\left(\tilde{b}^{T}-\mathbf{e}_{s}^{T} \tilde{A}\right) \partial_{x x}^{2} p^{n+1} \mathbf{e} \\
\overline{\mathbf{Q}}= & \Delta t \partial_{x}\left(\tilde{A}^{T} \mathbf{P}\right)+\Delta t\left(\tilde{b}^{T}-\mathbf{e}_{s}^{T} \tilde{A}\right) \partial_{x} p^{n+1} \mathbf{e}
\end{aligned}
$$

The latter are the adjoint equations (2.6) to problem (2.5) provided an implicit stiffly accurate scheme (2.9) has been used. Therein $\rho^{n+1}=\rho^{n}-\Delta t \tilde{b}^{T}\left(\partial_{x} \mathbf{J}+\partial_{x x}^{2} \mathbf{R}\right)+\Delta t b^{T}\left(\partial_{x x}^{2} \mathbf{R}\right)$ 
becomes $\rho^{n+1}=\mathbf{e}_{s} \mathbf{R}-\Delta t\left(\tilde{b}^{T}-\mathbf{e}_{s} \tilde{A}\right)\left(\partial_{x} \mathbf{J}+\partial_{x x}^{2} \mathbf{R}\right)$, which yields further simplifications in (2.8) and (2.10), respectively.

A particular, yet important case of Lemma 2.1 are the so-called globally stiffly accurate IMEX scheme. They fulfil additionally $\left(\tilde{b}^{T}-\mathbf{e}_{s}^{T} \tilde{A}\right)=0$.

\section{Optimal choice of $\mathcal{M}$}

In the following section we discuss the optimal choice of $\mu$ in equation (2.1). We want to avoid parabolic stiffness for small value of $\varepsilon$, and the numerical instabilities due to the discretization of the term $\left(j+\mu \rho_{x}\right)_{x}$. In [11] the following formula has been used $\mu=\exp (-\varepsilon / \Delta x)$, here we want to choose $\mu$ in such a way that Chapman-Enskog expansion with respect to $\varepsilon$ at least to order $O\left(\varepsilon^{2}\right)$ and the term $j+\mu \rho_{x}$ vanishes. It can been shown that independent of $\mu$ a stiffly accurate asymptotic-preserving IMEX yields an asymptoticpreserving scheme for the limit equation.

Considering an $s$-stage IMEX scheme and a semi-discretization of (1.5) as in (2.2), the optimal choice of an diagonal matrix $\mathcal{M}$, such that the explicit term $\mathbf{J}+\mathcal{M} \partial_{x} \mathbf{R}$ vanishes in the $O\left(\varepsilon^{2}\right)$ regime is presented in the following lemma.

Lemma 3.1. If the IMEX scheme is of type $A$ an optimal choice for $\mathcal{M}$ in the $O\left(\varepsilon^{2}\right)$ regime for scheme

$$
\begin{aligned}
\mathbf{R} & =\rho^{n} \mathbf{e}-\Delta t \tilde{A} \partial_{x}\left(\mathbf{J}+\mathcal{M} \partial_{x} \mathbf{R}\right)+\Delta t A \mathcal{M} \partial_{x x}^{2} \mathbf{R} \\
\varepsilon^{2} \mathbf{J} & =\varepsilon^{2} j^{n} \mathbf{e}-\Delta t A\left(\partial_{x} \mathbf{R}+\mathbf{J}\right) \\
\rho^{n+1} & =\rho^{n}-\Delta t \tilde{b}^{T} \partial_{x}\left(\mathbf{J}+\mathcal{M} \partial_{x} \mathbf{R}\right)+\Delta t b^{T} \mathcal{M} \partial_{x x}^{2} \mathbf{R} \\
\varepsilon^{2} j^{n+1} & =\varepsilon^{2} j^{n}-\Delta t b^{T}\left(\partial_{x} \mathbf{R}+\mathbf{J}\right)
\end{aligned}
$$

is given by

$$
\mathcal{M}=\Delta t\left(\varepsilon^{2} I d+\Delta t \operatorname{diag}(A)\right)^{-1} \operatorname{diag}(A) .
$$

The formula follows straightforward substituting stage by stage the approximation of order $O\left(\varepsilon^{2}\right)$ in the subsequent stages

$$
\begin{aligned}
J_{1} & =-\frac{a_{11} \Delta t}{\varepsilon^{2}+a_{11} \Delta t} \partial_{x} R_{1}+O\left(\varepsilon^{2}\right), \\
J_{2} & =-\frac{a_{22} \Delta t}{\varepsilon^{2}+a_{22} \Delta t} \partial_{x} R_{2}-\frac{a_{21} \Delta t}{\varepsilon^{2}+a_{22} \Delta t} \underbrace{\left(\partial_{x} R_{1}+J_{1}\right)}_{O\left(\varepsilon^{2}\right)}+O\left(\varepsilon^{2}\right)=-\frac{a_{22} \Delta t}{\varepsilon^{2}+a_{22} \Delta t} \partial_{x} R_{2}+O\left(\varepsilon^{2}\right) \\
& \vdots \\
J_{i} & =-\frac{a_{i i} \Delta t}{\varepsilon^{2}+a_{i i} \Delta t} \partial_{x} R_{i}-\sum_{j=1}^{i-1} \frac{a_{i j} \Delta t}{\varepsilon^{2}+a_{i i} \Delta t} \underbrace{\left(\partial_{x} R_{j}+J_{j}\right)}_{O\left(\varepsilon^{2}\right)}+O\left(\varepsilon^{2}\right)=-\frac{a_{i i} \Delta t}{\varepsilon^{2}+a_{i i} \Delta t} \partial_{x} R_{i}+O\left(\varepsilon^{2}\right) .
\end{aligned}
$$


We leave a rigorous proof in Appendix B.

Remark 1. Note that $\mathcal{M}=\operatorname{diag}\left(\mu_{j}^{n}\right)$ is not depending on $t_{n}$, i.e, $\mu_{j}^{n} \equiv \mu_{j}$, and the solution of (3.2) can be computed for the stages once and for all. Moreover (3.2) tells us that when $\varepsilon \rightarrow 0, \mathcal{M}$ has the expected behavior, namely $\mathcal{M} \rightarrow$ Id.

\section{Numerical results}

For the temporal discretization we use different IMEX schemes fulfilling the properties of Lemma 2.1. We consider second-order in time schemes. The IMEX GSA $(3,4,2)$, [30], as given by the Butcher tables in table 4 is a globally stiffly accurate scheme which is of type A. The implicit part is invertible and the last row of implicit and explicit scheme coincide. It is of second-order as the numerical results show. Further, we consider the second-order IMEX $\operatorname{SSP}(3,3,2)$ scheme, [11], (table 5) which is only implicitly stiffly accurate and of type A. In view of Theorem 3.1[30] we observe that $\operatorname{SSP}(3,3,2)$ is symplectic. Theorem 2.1[30] guarantees that for all considered schemes the convergence order of the IMEX scheme applied to the optimality system is also of second-order.

For the spatial discretization we introduce an equidistant grid with $M$ grid points $\left\{x_{i}\right\}_{i=1}^{M}$ and grid size $\Delta x$, such that $x_{1}=\frac{\Delta x}{2}$ and $x_{M}=1-\frac{\Delta x}{2}$. We set $\rho^{n}\left(x_{i}\right)=\rho_{i}^{n}$ and $j^{n}\left(x_{i}\right)=j_{i}^{n}$.

Since the Goldstein-Taylor model depends on $\varepsilon$, we expect parabolic behavior for $\varepsilon \ll 1$ and hyperbolic behavior else. We use second order central difference for the diffusive part $\rho_{x x}$ and hyperbolic discretization based on an Upwind scheme for the advective terms. In order to determine the Upwind direction, we recall from section 1 the definition of the macroscopic variables

$$
\rho=f^{+}+f^{-}, \quad j=\frac{1}{\varepsilon}\left(f^{+}-f^{-}\right) .
$$

We obtain for $f^{+}$, the density of particles with positive velocity, the Upwind scheme,

$$
\frac{f_{i}^{+}-f_{i-1}^{+}}{\Delta x}=\frac{f_{i+1}^{+}-f_{i-1}^{+}}{2 \Delta x}-\frac{\Delta x}{2} \frac{f_{i+1}^{+}-2 f_{i}^{+}-f_{i-1}^{+}}{(\Delta x)^{2}} .
$$

Similar for the scheme of $f^{-}$. By combining the discretization for $f^{+}$and $f^{-}$we obtain the discrete stencils in the original variables by applying (4.1), as follows:

$$
D^{h} \rho=D^{c} \rho-\frac{\varepsilon \Delta x}{2} D^{2} j, \quad D^{h} j=D^{c} j-\frac{\Delta x}{2 \varepsilon} D^{2} \rho
$$

where $D^{c}$ is the stencil for central difference $\frac{1}{\Delta x}\left(\begin{array}{lll}-1 & 0 & 1\end{array}\right)$ and $D^{2}$ the second order central difference $\frac{1}{(\Delta x)^{2}}(1-21)$. Using a convex combination of the discretization of the diffusive term with the hyperbolic part by the function $\Phi=\Phi(\varepsilon)$ we finally obtain

$$
D \rho=\Phi D^{c} \rho+(1-\Phi) D^{h} \rho, \quad D j=\Phi D^{c} j+(1-\Phi) D^{h} j
$$


The function $\Phi$ is chosen such that $\Phi(0)=1$ and $\Phi(\varepsilon) \frac{\Delta x}{2 \varepsilon} \rightarrow 0$ for $\varepsilon \rightarrow 1$. The simplest possible way is $\Phi=1-\varepsilon$, but more cleaver choices have been proposed in [10], where the value of $\Phi$ coincides with $\mu=\exp (-\varepsilon / \Delta x)$ or in [33] with $\Phi=1-\tanh (\varepsilon / \Delta x)$.

In all cases we discretize the with a spatial grid size $\Delta x \approx \Delta t$ since we avoid the parabolic CFL condition due to introduced splitting, (2.1). The discretization of $\Delta x \approx \Delta t$ is the typical hyperbolic CFL type condition induced by the transport.

\subsection{Order analysis}

To verify the theoretical results numerically we set up the following test problem. We consider the parabolic case. Let $\varepsilon=0, \nu=0, u_{l}=-1$ and $u_{r}=1$. Further set $\rho_{0}=\cos (x)$, $j_{0}=0$ and $\rho_{d}(x)=e^{-T} \cos (x)$. Then, the solution to the optimal control problem (1.4)(1.5) is given analytically by $u^{*}(t)=e^{-t}(\cos (1)-\sin (1))$ and $J=0$. Within this setting $\rho(x, t)=e^{-t} \cos (x)$ is solution of $(1.5)$ and $p^{*}(t, 1)=0$. The domain is $\Omega=[0,1]$ and the terminal time $T=1$.

We compute the numerical solution for different values of $N \in\{20,40,80,160,320\}$ using different IMEX schemes. We denote by $\rho_{N}^{*}$ and $p_{N}^{*}$ the solution to (2.3) with initial values $\rho_{d}=\rho(x, T)$ and $\rho^{N}=\rho_{N}^{*}$. We compare ratios of $L^{\infty}$ and $L^{1}$ errors of the approximate solutions using the following norms:

$$
L^{\infty}\left(L^{1}(\Omega)\right):=L^{\infty}\left(0, T ; L^{1}(\Omega)\right) \quad \text { and } \quad L^{\infty}\left(L^{\infty}(\Omega)\right):=L^{\infty}\left(0, T ; L^{\infty}(\Omega)\right) .
$$

The results for different IMEX schemes are listed in table 1 and 2.

As expected we observe the convergence order of two for all discussed schemes. We tested the example for stiffly accurate (SSP2(3,3,2)) as well as globally stiffly accurate schemes $(\operatorname{GSA}(3,4,2))$. The order two is in particular preserved in the limit $\varepsilon=0$ as expected by the previous Lemmas.

\begin{tabular}{c||c|c||l|l}
$N$ & $\left\|\rho_{N}^{*}-\rho_{d}\right\|_{L^{\infty}\left(L^{1}(\Omega)\right)}$ & $\left\|\rho_{N}^{*}-\rho_{d}\right\|_{L^{\infty}\left(L^{\infty}(\Omega)\right)}$ & $\left\|p_{N}^{*}\right\|_{L^{\infty}\left(L^{1}(\Omega)\right)}$ & $\left\|p_{N}^{*}\right\|_{L^{\infty}\left(L^{\infty}(\Omega)\right)}$ \\
\hline \hline 20 & $1.31 \mathrm{e}-04$ & $2.69 \mathrm{e}-07$ & $1.25 \mathrm{e}-04$ & $2.02 \mathrm{e}-07$ \\
40 & $3.10 \mathrm{e}-05(2.08)$ & $6.08 \mathrm{e}-08(2.14)$ & $3.03 \mathrm{e}-05(2.04)$ & $4.88 \mathrm{e}-08(2.04)$ \\
80 & $7.55 \mathrm{e}-06(2.04)$ & $1.43 \mathrm{e}-08(2.09)$ & $7.46 \mathrm{e}-06(2.02)$ & $1.21 \mathrm{e}-08(2.01)$ \\
160 & $1.86 \mathrm{e}-06(2.01)$ & $3.45 \mathrm{e}-09(2.05)$ & $1.85 \mathrm{e}-06(2.01)$ & $3.01 \mathrm{e}-09(2.00)$ \\
320 & $4.62 \mathrm{e}-07(2.00)$ & $8.41 \mathrm{e}-10(2.03)$ & $4.61 \mathrm{e}-07(2.00)$ & $7.55 \mathrm{e}-10(1.99)$ \\
\hline \hline
\end{tabular}

Table 1: Order results for the $\operatorname{GSA}(3,4,2)$, table $4, \varepsilon=0$. In brackets the the $\log _{2}$-ratio between the results from two subsequent step width. 


\begin{tabular}{c||c|c||l|l}
$N$ & $\left\|\rho_{N}^{*}-\rho_{d}\right\|_{L^{\infty}\left(L^{1}(\Omega)\right)}$ & $\left\|\rho_{N}^{*}-\rho_{d}\right\|_{L^{\infty}\left(L^{\infty}(\Omega)\right)}$ & $\left\|p_{N}^{*}\right\|_{L^{\infty}\left(L^{1}(\Omega)\right)}$ & $\left\|p_{N}^{*}\right\|_{L^{\infty}\left(L^{\infty}(\Omega)\right)}$ \\
\hline \hline 20 & $1.29 \mathrm{e}-04$ & $2.73 \mathrm{e}-07$ & $1.22 \mathrm{e}-04$ & $1.96 \mathrm{e}-07$ \\
40 & $3.07 \mathrm{e}-05(2.06)$ & $6.15 \mathrm{e}-08(2.15)$ & $2.99 \mathrm{e}-05(2.03)$ & $4.80 \mathrm{e}-08(2.02)$ \\
80 & $7.51 \mathrm{e}-06(2.03)$ & $1.44 \mathrm{e}-08(2.09)$ & $7.42 \mathrm{e}-06(2.02)$ & $1.19 \mathrm{e}-08(2.00)$ \\
160 & $1.85 \mathrm{e}-06(2.01)$ & $3.46 \mathrm{e}-09(2.05)$ & $1.85 \mathrm{e}-06(2.00)$ & $3.00 \mathrm{e}-09(1.99)$ \\
320 & $4.62 \mathrm{e}-07(2.00)$ & $8.43 \mathrm{e}-10(2.03)$ & $4.61 \mathrm{e}-07(2.00)$ & $7.52 \mathrm{e}-10(1.99)$ \\
\hline \hline
\end{tabular}

Table 2: Order results for the SSP2 $(3,3,2)$, table $5, \varepsilon=0$. In brackets the $\log _{2}$-ratio between the results from two subsequent step width corresponds.

\subsection{Computational results on the optimal control problem}

We compare the IMEX methods applied to the Goldstein-Taylor model in the limit case $\varepsilon=0$ with the numerical solution presented in [39]. Therein, the limit problem has been studied using parameters: $T=1.58, \rho_{o}=j_{0}=0, \rho_{d}(x)=0.5\left(1-x^{2}\right), \nu=0.001, u_{l}=-1$ and $u_{r}=1$. We furthermore set $N=100$ and $M=50$. We use a gradient based optimization to iteratively compute the optimal control $u^{*}$ using an implicit stiffly accurate scheme (ISA). The numerical approximation to the gradient for the reduced objective functional $\tilde{J}(u)$ is then given by

$$
\nabla \tilde{J}=\Delta t\left(\nu u^{n}+p^{n}\right)
$$

where $p^{n}$ is the solution to the adjoint equation (2.3), respectively (2.8), at time $t^{n}$. The terminal condition for the gradient based optimization is $\left\|\operatorname{proj}_{\left[u_{l}, u_{r}\right]}(\nabla \tilde{J})\right\|_{L^{2}(0, T)} \leq 10^{-6}$.

The final values for $J\left(u_{\varepsilon}^{*}\right)$ for the different schemes are presented in table 3 . The calculated values with our method $J\left(u_{0}^{*}\right)$ are consistent with respect to the numerical discretization in space and time to the ones in [39]. Note that in the limit $\varepsilon=0$ we do not have a parabolic CFL condition due to the applied splitting and the obtained results are precisely as in [39].

\begin{tabular}{c||c|c|c|c|c} 
IMEX & $\varepsilon=0$ & $\varepsilon=0.1$ & $\varepsilon=0.5$ & $\varepsilon=0.8$ & $\varepsilon=1$ \\
\hline \hline $\operatorname{GSA}(3,4,2)$ & $6.51 \cdot 10^{-4}$ & $5.94 \cdot 10^{-4}$ & $2.85 \cdot 10^{-4}$ & $2.47 \cdot 10^{-4}$ & $2.44 \cdot 10^{-4}$ \\
\hline $\operatorname{SSP}(3,3,2)$ & $6.52 \cdot 10^{-4}$ & - & $2.84 \cdot 10^{-4}$ & $2.46 \cdot 10^{-4}$ & $2.43 \cdot 10^{-4}$ \\
\hline \hline
\end{tabular}

Table 3: Results for $J\left(u_{\varepsilon}^{*}\right)$, different IMEX schemes and values of $\varepsilon$. For $\varepsilon=0$ in [39], they obtain $J(u)=6.86 \cdot 10^{-4}$.

Figure 1 shows the numerical solutions using GSA $(3,4,2)$ scheme for different values of $\varepsilon \in\{0,0.1,0.5,1\}$. The globally stiffly accurate IMEX schemes yield solutions to the $\varepsilon$-dependent class of optimization problems (1.5) across the full range of parameters $\varepsilon$. 

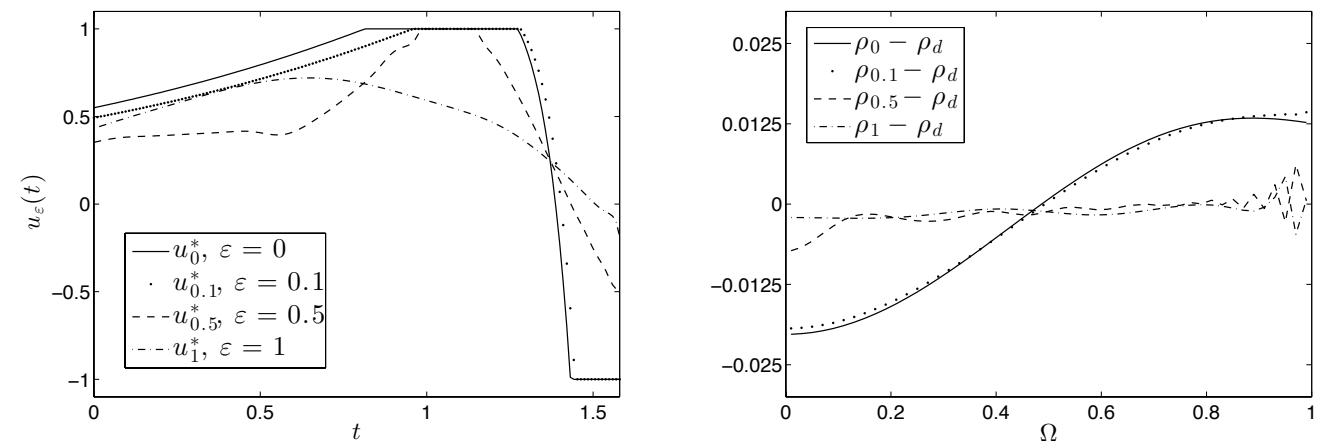

Figure 1: Numerical solution, using GSA(3,4,2) scheme, see appendix A, for 150 time steps and 50 grid points in space. The left part of the plot shows the optimal controls $u_{\varepsilon}^{*}$ for different values of $\varepsilon$. On the right plot we show the corresponding optimal states $\rho_{\varepsilon}^{*}(\cdot, T)-\rho_{d}$ for different choices of $\varepsilon$.

In figure 2 we plot the numerical solutions using $\operatorname{SSP}(3,3,2)$ scheme for different values of $\varepsilon \in\{0,0.5,0.8,1\}$. As in [11] shown, $\operatorname{SSP}(3,3,2)$ is not globally stiffly accurate, and therefore we cannot expect stability for small values of $\varepsilon$, even if $\varepsilon=0$ provides a stable solution. Further, we set $N=200$ for similar reasons.
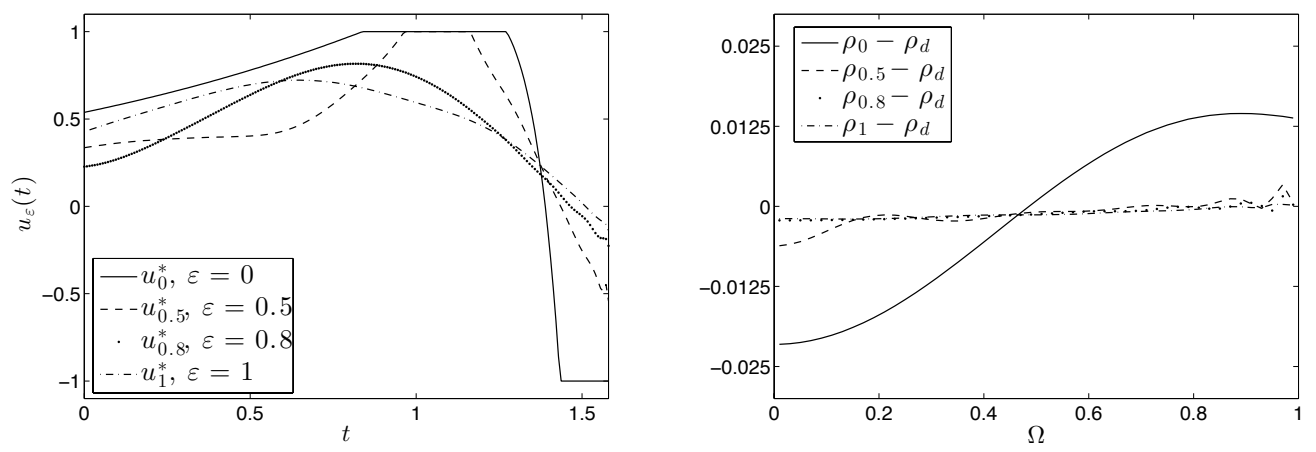

Figure 2: Numerical solution, using SSP2(3,3,2), i.e. table 5, for 200 time steps and 50 grid points in space. On the left the optimal control $u^{*}$ is plotted. The right part shows the difference of the optimal state to the desired state, i.e. $\rho_{\varepsilon}^{*}(\cdot, T)-\rho_{d}$.

In both figures, one can observe oscillations at the boundary $x=1$ for values of $\varepsilon>0.25$. This is caused due to the assumption $j=-\rho_{x}$ in $x=1$, which holds true just for $\varepsilon=0$. We set $\Phi=0.3$ for $\operatorname{GSA}(3,4,2)$ and $\varepsilon=1$. Further for $\operatorname{SSP}(3,3,2)$ and $\varepsilon=0.5$ we set $\Phi=0.385$. All other values of $\varepsilon$ are treated with $\Phi=1-\varepsilon^{3}$. 


\section{A Definitions of implicit-explicit Runge-Kutta methods}

We consider the Cauchy problem for a system of ODEs such that

$$
y^{\prime}=f(y)+g(y), \quad y(0)=y_{0}, \quad t \in[0, T],
$$

where $y(t) \in \mathbb{R}$ and $f, g: \mathbb{R} \longrightarrow \mathbb{R}$ Lipschitz continuous functions. Using an Implicit-Explict Runge-Kutta method with time step $\Delta t$ we obtain the following numerical scheme for (A.1)

$$
\begin{aligned}
& \mathbf{Y}=y^{n} \mathbf{e}+\Delta t(\tilde{A} \mathbf{F}(\mathbf{Y})+A \mathbf{G}(\mathbf{Y})) \\
& y^{n+1}=y^{n}+\Delta t\left(\tilde{b}^{T} \mathbf{F}(\mathbf{Y})+b^{T} \mathbf{G}(\mathbf{Y})\right)
\end{aligned}
$$

where $\mathbf{Y}=\left(Y_{l}(\cdot)\right)_{l=1}^{s}$ denotes the $s$ stage variables, and $\mathbf{F}\left(\mathbf{Y}^{n}\right)=\left(f\left(Y_{l}\right)\right)_{l=1}^{s}, \mathbf{G}(\mathbf{Y})=$ $\left(g\left(Y_{l}\right)\right)_{l=1}^{s}$, moreover $\mathbf{e}=(1, \ldots, 1) \in \mathbb{R}^{s}$. The matrices $\tilde{A}, A$ are $s \times s$ matrices, and $\tilde{c}, c, \tilde{b}, b \in$ $\mathbb{R}^{s}$. We take in account IMEX schemes satisfying the following definition

Definition A.1. A diagonally implicit IMEX Runge Kutta (DIRK) method is such that matrices $\tilde{A}$, and $A$ are lower triangular, where $\tilde{A}$ has zero diagonal.

Further we consider the following basic assumptions on $\tilde{c}, c, \tilde{b}, b \in \mathbb{R}^{s}$

$$
\sum_{i=1}^{s} b_{i}=1, \quad \sum_{i=1}^{s} \tilde{b}_{i}=1, \quad \tilde{c}_{i}=\sum_{j=1}^{i-1} \tilde{a}_{i j}, \quad c_{i}=\sum_{j=1}^{i} a_{i j}
$$

Those conditions need to be fulfilled for a first order Runge Kutta method. Increasing the order of a Runge Kutta method increases the number of restrictions on the coefficients in the Butcher tables. For IMEX methods up to order $k=3$, the number of constraints can be reduced if $c=\tilde{c}$ and $b=\tilde{b}[38,37]$.

Definition A.2 (Type A $[11,35])$. If $A$ is invertible the IMEX scheme is of type A.

\begin{tabular}{c|cccc}
0 & 0 & 0 & 0 & 0 \\
$3 / 2$ & $3 / 2$ & 0 & 0 & 0 \\
$1 / 2$ & $5 / 6$ & $-1 / 3$ & 0 & 0 \\
1 & $1 / 3$ & $1 / 6$ & $1 / 2$ & 0 \\
\hline & $1 / 3$ & $1 / 6$ & $1 / 2$ & 0
\end{tabular}

\begin{tabular}{c|cccc}
$1 / 2$ & $1 / 2$ & 0 & 0 & 0 \\
$5 / 4$ & $3 / 4$ & $1 / 2$ & 0 & 0 \\
$1 / 4$ & $-1 / 4$ & 0 & $1 / 2$ & 0 \\
1 & $1 / 6$ & $-1 / 6$ & $1 / 2$ & $1 / 2$ \\
\hline & $1 / 6$ & $-1 / 6$ & $1 / 2$ & $1 / 2$
\end{tabular}

Table 4: GSA(3,4,2), [30], Type A scheme and globally stiffly accurate, (GSA). 
Definition A.3 (Type GSA [11]). An IMEX method is globally stiffly accurate (GSA), if $\tilde{c}_{s}=c_{s}=1$ and

$$
\tilde{b}^{T}=\mathbf{e}_{s}^{T} \tilde{A} \quad \text { and } \quad b^{T}=\mathbf{e}_{s}^{T} A,
$$

If the previous equalities hold only for the implicit part, the method is implicit stiffly accurate (ISA).

To denote each IMEX scheme we use the following convention for the names of the schemes: $\operatorname{Acronym}\left(\sigma_{E}, \sigma_{I}, k\right)$, where $\sigma_{E}$ denoting the effective number of stages of the explicit, $\sigma_{I}$ of the implicit scheme. and $k$ the combined order of accuracy.

\begin{tabular}{|c|c|c|c|c|c|c|c|}
\hline 0 & 0 & 0 & 0 & $1 / 4$ & $1 / 4$ & 0 & 0 \\
\hline $1 / 2$ & $1 / 2$ & 0 & 0 & $1 / 4$ & 0 & $1 / 4$ & 0 \\
\hline 1 & $1 / 2$ & $1 / 2$ & 0 & 1 & $1 / 3$ & $1 / 3$ & $1 / 3$ \\
\hline & $1 / 3$ & $1 / 3$ & $1 / 3$ & & $1 / 3$ & $1 / 3$ & $1 / 3$ \\
\hline
\end{tabular}

Table 5: SSP2(3,3,2) [35], Type A and implicit stiffly accurate scheme (ISA).

\section{B Proof of Lemma 3.1}

Let consider system (3.1), we can decompose matrix $A$ in this way $A=D+L$, where $D=\operatorname{diag}(A)$ and $L$ is the lower triangular part of $A$, Therefore we can rewrite the second equation for $\mathbf{J}$ in this way

$$
\begin{aligned}
\varepsilon^{2} \mathbf{J} & =\varepsilon^{2} j^{n} \mathbf{e}-\Delta t D\left(\partial_{x} \mathbf{R}+\mathbf{J}\right)-\Delta t L\left(\partial_{x} \mathbf{R}+\mathbf{J}\right) \\
\left(\varepsilon^{2} I d+\Delta t D\right) \mathbf{J} & =\varepsilon^{2} j^{n} \mathbf{e}-\Delta t D \partial_{x} \mathbf{R}-\Delta t L\left(\partial_{x} \mathbf{R}+\mathbf{J}\right)
\end{aligned}
$$

Neglecting the $O\left(\varepsilon^{2}\right)$ term and inverting the diagonal matrix on the lefthand side we have

$$
\mathbf{J}=-\underbrace{\Delta t\left(\varepsilon^{2} I d+\Delta t D\right)^{-1} D}_{\mathcal{M}} \partial_{x} \mathbf{R}-\underbrace{\Delta t\left(\varepsilon^{2} I d+\Delta t D\right)^{-1} L}_{\mathcal{K}}\left(\partial_{x} \mathbf{R}+\mathbf{J}\right)+o\left(\varepsilon^{2}\right) .
$$

Recursively we substitute in $J$ (B.1) itself, the first recursion gives

$$
\mathbf{J}=-\mathcal{M} \partial_{x} \mathbf{R}+\mathcal{K}(I d-\mathcal{M}) \partial_{x} \mathbf{R}+\mathcal{K}^{2}\left(\partial_{x} \mathbf{R}+\mathbf{J}\right)+o\left(\varepsilon^{2}\right)
$$

applying the recursion $s-1$ times we obtain

$$
\begin{aligned}
\mathbf{J} & =-\mathcal{M} \partial_{x} \mathbf{R}-\sum_{l=1}^{s-1}(-\mathcal{K})^{l}(I d-\mathcal{M}) \partial_{x} \mathbf{R}-(-\mathcal{K})^{s}\left(\partial_{x} \mathbf{R}+\mathbf{J}\right)+o\left(\varepsilon^{2}\right)= \\
& =-\mathcal{M} \partial_{x} \mathbf{R}+\left(\sum_{l=1}^{s-1}(-1)^{l-1} \mathcal{K}^{l}\right)(I d-\mathcal{M}) \partial_{x} \mathbf{R}+o\left(\varepsilon^{2}\right),
\end{aligned}
$$


where in the last equation $\mathcal{K}^{s}$ vanishes since it is a nilpotent matrix of grade $s$, moreover each element of matrix $I d-\mathcal{M}$ has order $o\left(\varepsilon^{2}\right)$, from a direct computation on the general $i$ element of the diagonal matrix we have

$$
(I d-\mathcal{M})_{i}=1-\frac{\Delta t a_{i i}}{\varepsilon^{2}+\Delta t a_{i i}}=1-\frac{\Delta t a_{i i}}{\varepsilon^{2}+\Delta t a_{i i}}=\frac{\varepsilon^{2}}{\varepsilon^{2}+\Delta t a_{i i}}
$$

Thus the expression for $\mathbf{J}$ reads

$$
\mathbf{J}=-\mathcal{M} \partial_{x} \mathbf{R}+o\left(\varepsilon^{2}\right)
$$

which cancel exactly the explicit part of the semi-discretize scheme, in the $o\left(\varepsilon^{2}\right)$ regime. Hence the appropriate choice for $\mathcal{M}$ is given by

$$
\mathcal{M}=\Delta t\left(\varepsilon^{2} I d+\Delta t D\right)^{-1} D
$$

In table 6 we show $\mathcal{M}$ for different schemes using the provided method. Note that we use the same $\mathcal{M}$ for the adjoint equations.

\begin{tabular}{|c|c|}
\hline IMEX & $\mathcal{M}(\varepsilon)$ \\
\hline $\operatorname{GSA}(3,4,2)$ & $\frac{\Delta t}{2 \varepsilon^{2}+\Delta t} \operatorname{diag}(1,1,1,1)$ \\
\hline $\operatorname{SSP} 2(3,3,2)$ & $\operatorname{diag}\left(\frac{\Delta t}{4 \varepsilon^{2}+\Delta t}, \frac{\Delta t}{4 \varepsilon^{2}+\Delta t}, \frac{\Delta t}{3 \varepsilon^{2}+\Delta t}\right)$ \\
\hline
\end{tabular}

Table 6: Optimal choice of matrix $\mathcal{M}$ for the different IMEX schemes used.

Acknowledgement This work has been supported by EXC128, DAAD 54365630, 55866082 .

\section{References}

[1] U. Ascher, S. Ruuth, and R. Spiteri, Implicit-explicit Runge-Kutta methods for time-dependent partial differential equations, Applied Numerical Mathematics, 25 (1997), 151-167

[2] M. Baines, M. Cullen, C. Farmer, M. Giles, and M. Rabbitt, eds., 8th ICFD Conference on Numerical Methods for Fluid Dynamics. Part 2, John Wiley \& Sons Ltd., Chichester, 2005. Papers from the conference held in Oxford, 2004, Internat. J. Numer. Methods Fluids 47 (2005), no. 10-11.

[3] M. K. Banda and M. Herty, Adjoint IMEX-based schemes for control problems governed by hyperbolic conservation laws, Comp. Opt. and App., Vol. 51(2), (2012), 090-930. 
[4] S. Bianchini, On the shift differentiability of the flow generated by a hyperbolic system of conservation laws, Discrete Contin. Dynam. Systems, 6 (2000), pp. 329-350.

[5] F. Bouchut And F. James, One-dimensional transport equations with discontinuous coeffcients, Nonlinear Anal., 32 (1998), pp. 891-933.

[6] - Differentiability with respect to initial data for a scalar conservation law, in Hyperbolic problems: theory, numerics, applications, Vol. I (Zürich, 1998), vol. 129 of Internat. Ser. Numer. Math., Birkhäuser, Basel, 1999, pp. 113-118.

[7] — Duality solutions for pressureless gases, monotone scalar conservation laws, and uniqueness, Comm. Partial Differential Equations, 24 (1999), pp. 2173-2189.

[8] F. Bouchut, F. JAMEs, And S. MANCini, Uniqueness and weak stability for multidimensional transport equations with one-sided Lipschitz coefficient, Ann. Sc. Norm. Super. Pisa Cl. Sci. (5), 4 (2005), pp. 1-25.

[9] J. F. Bonnans and J. Laurent-Varin, Computation of order conditions for symplectic partitioned Runge-Kutta schemes with application to optimal control, Numerische Mathematik, 103 (2006), $1-10$.

[10] S. Boscarino, P.G. LeFloch, G. Russo, High-Order asymptotic-preserving methods for fully nonlinear relaxation problems, submitted to SIAM Journal on Scientific Computing (SISC).

[11] S. Boscarino, L. Pareschi, and G. Russo. Implicit-explicit runge-kutta schemes for hyperbolic systems and kinetic equations in the diffusion limit. SIAM J. Scient. Comp., 35(1):A22-A51, 2013.

[12] A. Bressan And G. Guerra, Shift-differentiability of the flow generated by a conservation law, Discrete Contin. Dynam. Systems, 3 (1997), pp. 35-58.

[13] A. Bressan And M. Lewicka, Shift differentials of maps in BV spaces, in Nonlinear theory of generalized functions (Vienna, 1997), vol. 401 of Chapman \& Hall/CRC Res. Notes Math., Chapman \& Hall/CRC, Boca Raton, FL, 1999, pp. 47-61.

[14] A. Bressan And A. Marson, A variational calculus for discontinuous solutions to conservation laws, Comm. Partial Differential Equations, 20 (1995), pp. 1491-1552.

[15] A. Bressan And W. Shen, Optimality conditions for solutions to hyperbolic balance laws, Control methods in PDE-dynamical systems, Contemp. Math., 426 (2007), pp. 129-152.

[16] M.H. Carpenter, C.A. Kennedy, Additive Runge-Kutta schemes for convection-diffusionreaction equations, Appl. Numer. Math. 44 (2003), no. 1-2, 139Đ181.

[17] C. Castro, F. Palacios, and E. Zuazua, An alternating descent method for the optimal control of the inviscid Burgers equation in the presence of shocks, Math. Models Methods Appl. Sci., 18 (2008), pp. 369-416.

[18] G. Dimarco and L. Pareschi, Asymptotic-Preserving IMEX Runge-Kutta methods for nonlinear kinetic equations, preprint, (2012) 
[19] A. L. Dontchev and W. W. Hager The Euler approximation in state constrained optimal control Math. Comp., 70 (2001), 173-203

[20] A. L. Dontchev and W. W. Hager and V. M. Veliov Second-order Runge-Kutta approximations in control constrained optimal control SIAM J. Numer. Anal., 38 (2000), 202-226

[21] M. Giles And S. UlBrich, Convergence of linearized and adjoint approximations for discontinuous solutions of conservation laws. Part 1: Linearized approximations and linearized output functionals, SIAM J. Numer. Anal., 48 (2010), pp. 882-904.

[22] — Convergence of linearized and adjoint approximations for discontinuous solutions of conservation laws. Part 2: Adjoint approximations and extensions, SIAM J. Numer. Anal., 48 (2010), pp. 905-921.

[23] M. B. GiLes, Analysis of the accuracy of shock-capturing in the steady quasi 1d-euler equations, Int. J. Comput. Fluid Dynam., 5 (1996), pp. 247-258.

[24] M. B. GILES, Discrete adjoint approximations with shocks, in Hyperbolic problems: theory, numerics, applications, Springer, Berlin, 2003, pp. 185-194.

[25] M. B. Giles And N. A. Pierce, Analytic adjoint solutions for the quasi-one-dimensional Euler equations, J. Fluid Mech., 426 (2001), pp. 327-345.

[26] - Adjoint error correction for integral outputs, in Error estimation and adaptive discretization methods in computational fluid dynamics, vol. 25 of Lect. Notes Comput. Sci. Eng., Springer, Berlin, 2003, pp. 47-95.

[27] M. B. Giles AND E. SÜLI, Adjoint methods for PDEs: a posteriori error analysis and postprocessing by duality, Acta Numer., 11 (2002), pp. 145-236.

[28] W. W. Hager, Runge-Kutta methods in optimal control and the transformed adjoint system, Numerische Mathematik, 87 (2000), 247-282.

[29] M. Herty and V. Schleper, Time discretizations for numerical optimization of hyperbolic problems, App. Math. Comp. 218 (2011), 183-194.

[30] M. Herty, L. Pareschi, and S. Steffensen. Implicit-explicit runge-kutta schemes for numerical discretization of optimal control problems. to appear, SIAM J. Num. Analysis, 2013

[31] I. Higueras, Strong stability for additive Runge-Kutta methods SIAM J. Num. Anal., 44 (2006), $1735-1758$.

[32] C. A. Kennedy and M. H. Carpenter Additive Runge-Kutta schemes for convection-diffusionreaction equations, Appl. Num. Math., 44 (2003), 139-181.

[33] S. Jin and C. D. Levermore, Numerical Schemes For Hyperbolic Conservation Laws With Stiff Relaxation Terms, J. Comput. Phys, 126 (1996), pp. 449-467.

[34] J. Lang and J. Verwer W-Methods in optimal control Preprint 2011, TU Darmstadt 
[35] L. Pareschi and G. Russo, Implicit-explicit Runge-Kutta schemes and applications to hyperbolic systems with relaxation, J. Sci. Comput., 25 (2005), 129-155.

[36] L. Pareschi and G. Russo, Implicit-explicit Runge-Kutta schemes for stiff systems of differential equations Recent Trends in Numerical Analysis, Edited by L.Brugnano and D.Trigiante, 3 (2000), 269-289.

[37] J. M. Sanz-Serna, Runge-Kutta Schemes for Hamiltonian Systems, BIT, 28 (1988), 877-883.

[38] J. M. Sanz-Serna and L. Abia, Order Conditions for Canonical Runge-Kutta Schemes, SIAM J. Numer. Anal., 28 (1991), 1081-1096.

[39] F. Tröltzsch and D. Wachsmuth. On convergence of a receding horizon method for parabolic boundary control. Optim. Methods Softw., 19(2):201-216, 2004.

[40] S. UlBRICH, Optimal control of nonlinear hyperbolic conservation laws with source terms, Habilitation Thesis, Technische Universitaet Muenchen, 2001.

[41] S. Ulbrich, Adjoint-based derivative computations for the optimal control of discontinuous solutions of hyperbolic conservation laws, Syst. Control Lett., 48 (2003), pp. 313-328.

[42] — On the superlinear local convergence of a filer-sqp method, Math. Prog. Ser. B, 100 (2004), pp. 217-245.

[43] A. Walther Automatic differentiation of explicit Runge-Kutta methods for optimal control J. Comp. Opt. Appl., 36 (2007), 83-108 